\title{
The Local Central Limit Theorem for a Gibbs Random Field
}

\author{
M. Campanino, D. Capocaccia and B. Tirozzi
}

Instituto di Matematica "G. Castelnuovo", University of Rome, and Instituto di Matematica, University of Aquila, Italy

\begin{abstract}
We extend the validity of the implication of a local limit theorem from an integral one. Our extension eliminates the finite range assumption present in the previous works by using the cluster expansion to analyze the contribution from the tail of the potential.
\end{abstract}

\section{Definitions and Results}

\subsection{Assumptions}

We consider a $v$-dimensional lattice spin system, to each $x \in \mathbb{Z}^{v}$ is associated a spin $s_{x}$ that can take all the integer values lying between the two integers $n, m$. We denote by $\sigma$ the $\max (|n|,|m|)$, A configuration $s_{\Lambda}$ in a subset $\Lambda, \Lambda \subset \mathbb{Z}^{v}$ is given by an element of $\{n, \ldots m\}\}^{\Lambda}$. If $\Lambda, M$ are two disjoint subsets of $\mathbb{Z}^{v}$, we denote by $s_{A} \vee s_{M}$ the spin configuration in $\Lambda \cup M$ individuated by $s_{A}, s_{M}$. The interaction is given by a pair long range potential of the form $J(x-y) s_{x} s_{y}$ where $J$ is a real function on $\mathbb{Z}^{v}$. We assume that:

$$
\sum_{t \in \mathbb{Z}^{v}}|J(t)|^{1 / 2}=\gamma<\infty
$$

1.2. Definition. Let $\Lambda$ be a finite subset of $\mathbb{Z}^{v}$. The Gibbs conditional distribution on the set of configurations in $\Lambda$, with condition $s_{\mathbb{Z} \backslash \Lambda}$ is defined as:

$$
p_{\Lambda}\left(s_{\Lambda} \mid s_{\mathbb{Z} \backslash \backslash \Lambda}\right)=\exp \left\{\sum_{\substack{x, y \in A \\ x \neq y}} J(x-y) s_{x} s_{y}+\sum_{x \in A} h_{x}\left(s_{x} \mid s_{\mathbb{Z}^{v} \backslash \Lambda}\right)\right\} / Z_{\Lambda}(h, J)
$$

where

$$
h_{x}\left(s_{x} \mid s_{\mathbb{Z}{ }^{\nu} \backslash \Lambda}\right)=\sum_{y \in \mathbb{Z} \backslash \backslash \Lambda} J(x-y) s_{x} s_{y}+J(0) s_{x}^{2}
$$

and $Z_{\Lambda}(h, J)$ is the partition function for the set of spins in $\Lambda$, with pair interaction $J$, 
and external field $h$ :

$$
Z_{\Lambda}(h, J)=\sum_{s_{\Lambda}} \exp \left\{\sum_{x, y \in \Lambda, x \neq y} J(x-y) s_{x} s_{y}+\sum_{x \in \Lambda} h_{x}\left(s_{x} \mid s_{\mathbb{Z}^{v} \backslash \Lambda}\right)\right\}
$$

1.3. Definition. We call a Gibbsian sequence a sequence of pairs $\left(p_{\infty}\left(\cdot \mid s_{\mathbb{Z} v \backslash \Lambda_{k}}^{k}\right)\right.$, $\left.s_{\mathbb{Z}^{v} \backslash \Lambda_{k}}^{k}\right)$ where $\Lambda_{k}$ is a sequence of cubes in $\mathbb{Z}^{v}$, such that $\Lambda_{k} \subset \Lambda_{k+1}$ and $\bigcup_{k=1}^{\infty} \Lambda_{k}=\mathbb{Z}^{v}$, and $s_{\mathbb{Z}^{v} \backslash \Lambda_{k}}^{k}$ is a sequence of configurations in $\mathbb{Z}^{v} \backslash \Lambda_{k}$. Let $E_{k}$ denote the expectation, and $P_{k}$ the probability computed by means of the probability distribution $p_{\Lambda_{k}}\left(\cdot \mid s_{\mathbb{Z}^{\nu} \backslash \Lambda_{k}}^{k}\right)$ and for each $k$, let $\xi_{k}, \bar{\xi}_{k}$ be the random variables

$$
\xi_{k}=\sum_{x \in \Lambda_{k}} s_{x} \quad \bar{\xi}_{k}=\left(\xi_{k}-E_{k}\left(\xi_{k}\right)\right) / \sqrt{D_{k}}
$$

where $D_{\dot{k}}=E_{k}\left(\left(\xi_{k}-E_{k}\left(\xi_{k}\right)\right)^{2}\right)$ denotes the variance of $\xi_{k}$.

1.4. Definitions. We say that the integral central limit theorem holds for the Gibbsian sequence $\left(p_{\Lambda_{k}}\left(\cdot \mid s_{\mathbb{Z}^{v} \backslash \Lambda_{k}}^{k}\right), s_{\mathbb{Z}^{\eta} \backslash \Lambda_{k}}^{k}\right)$ if:

(i) $\lim _{k \rightarrow \infty} D_{k} /\left|\Lambda_{k}\right|=\alpha$

(ii) $\alpha>0$

(iii) $\lim _{k \rightarrow \infty} P_{k}\left\{\bar{\xi}_{k} \leqq x\right\}=\int_{-\infty}^{x} \exp \left(-z^{2} / 2\right) d z / \sqrt{2 \pi}$

We say that the local central limit theorem holds for the Gibbsian sequence $\left(p_{\Lambda_{k}}\left(\cdot \mid s_{\mathbb{Z}^{v} \backslash \Lambda_{k}}^{k}\right), s_{\mathbb{Z}^{v} \backslash \Lambda_{k}}^{k}\right)$ if (i), (ii) are verified and

$$
\lim _{k \rightarrow \infty} \sup _{p}\left|\sqrt{D_{k}} P_{k}\left\{\xi_{k}=p\right\}-\exp \left(-\left(p-E_{k}\left(\xi_{k}\right)\right)^{2} / 2 D_{k}\right) / \sqrt{2 \pi}\right|=0 .
$$

The aim of this paper is the proof of the following:

1.5. Theorem. Under the assumption 1.1, if the integral central limit theorem holds for a given Gibbsian sequence, then the local central limit theorem holds for the same Gibbsian sequence.

The method of the proof follows the one introduced for independent random variables [1], and extended to lattice spin systems with short range interaction $[2,3]$. We briefly describe it in Sect. 2, where we reduce the proof of Theorem 1.5 to estimates for the characteristic function of a sum of "almost independent" random variables. These estimates are obtained in Sect. 3, expressing the characteristic function as a ratio of partition functions of suitable "polymer models" with hard core interaction, that can be estimated by a cluster expansion.

Our result can be extended to more general lattice spin systems, including the case of more general two body and many body potentials; assumption (1.1) on two body potential can also be weakened, and we limit the proof to the model described above for the sake of simplicity.

\section{Method of the Proof}

In this section we show the line of the proof of Theorem 1.5. Let $r_{0}$ be an integer, and $\mathbb{Z}^{v}\left(r_{0}\right)$ the sublattice of $\mathbb{Z}^{v}$ defined by the set of $v$-ples $\left\{n_{1} r_{0}, \ldots, n_{v} r_{0}\right.$; 
$\left.n_{i} \in \mathbb{Z}, i=1, \ldots, v\right\}$. With the notation of Sect. 1, let $\tilde{\Lambda}_{k}=\Lambda_{k} \cap \mathbb{Z}^{v}\left(r_{0}\right)$ and $\tilde{E}_{k}\left(\cdot \mid s_{\Lambda_{k} \backslash \tilde{A}_{k}}\right)$ denotes the expectation with respect to the probability distribution $p_{\tilde{\Lambda}_{k}}\left(\cdot \mid s_{\Lambda_{k} \backslash \tilde{\Lambda}_{k}} \vee s_{\mathbb{Z}^{\eta} \backslash \Lambda_{k}}^{k}\right)$. The characteristic function $f_{k}=E_{k}\left(\exp \left(i t \bar{\xi}_{k}\right)\right)$ satisfies the inequality:

$$
\begin{aligned}
\left|f_{k}(t)\right| & =\left|E_{k}\left(\tilde{E}_{k}\left(\exp \left(i t \bar{\xi}_{k}\right) \mid s_{\Lambda_{k} \backslash \tilde{\Lambda}_{k}}\right)\right)\right| \\
& \leqq \sup _{s_{\Lambda_{k} \backslash \tilde{\Lambda}_{k}}}\left|\widetilde{E}_{k}\left(\exp \left(\left(i t \sum_{x \in \tilde{\Lambda}_{k}} s_{x}\right) / \sqrt{D_{k}}\right) \mid s_{\Lambda_{k} \backslash \tilde{\Lambda}_{k}}\right)\right|
\end{aligned}
$$

If $r_{0}$ is large enough, the spins in $\tilde{\Lambda}_{k}$ are "almost not interacting", and one can expect that the r.h.s. of (2.1) behaves as the characteristic function for a sum of independent random variables. This is indeed the meaning of the following lemmas. We assume that 1.1 holds.

2.2. Lemma. There is a positive constant $D$, not depending on $s_{\mathbb{Z}^{\nu} \backslash \Lambda_{k}}^{k}$, such that, if $\delta, r_{0}^{-1}$ are small enough and $|t| \leqq \delta \sqrt{D_{k}}$, then:

$$
\left|\tilde{E}_{k}\left(\exp \left(i t \sum_{x \in \tilde{\Lambda}_{k}} s_{x} / \sqrt{D_{k}}\right) \mid s_{\Lambda_{k} \backslash \tilde{\Lambda}_{k}}\right)\right| \leqq \exp \left(-t^{2}\left|\tilde{\Lambda}_{k}\right| D / D_{k}\right)
$$

uniformly with respect to $S_{\Lambda_{k} \backslash \tilde{\Lambda}_{k}}$.

2.3. Lemma. For every finite $\delta$, there is a positive constant $C$, not depending on the sequence $s_{\mathbb{Z}^{v} \backslash \Lambda_{k}}^{k}$, such that, if $\delta \sqrt{D_{k}} \leqq|t| \leqq \pi \sqrt{D_{k}}$, and $r_{0}$ is large enough, then

$$
\left|\tilde{E}_{k}\left(\exp \left(i t \sum_{x \in \tilde{\Lambda}_{k}} s_{x} / \sqrt{D_{k}}\right) \mid s_{\Lambda_{k} \backslash \tilde{\Lambda}_{k}}\right)\right| \leqq \exp \left(-c\left|\tilde{\Lambda}_{k}\right|\right)
$$

uniformly with respect to $s_{\Lambda_{k} \backslash \tilde{\Lambda}_{k}}$.

In [2] it was sufficient to take $r_{0}$ bigger than the range of the potential in (2.2), (2.3), to have them satisfied. In the case of long range potential we cannot have the conditional independence of the random variables $s_{x}, x \in \tilde{\Lambda}_{k}$, under the conditions $S_{\Lambda_{k} \backslash \tilde{\Lambda}_{k}}$, but we can show, using a cluster expansion, that the long tail of the potential causes the addition of a term $\varphi\left(r_{0}\right)$ in the arguments of the exponentials in the r.h.s. of (2.2), (2.3) which becomes infinitesimal if $r_{0}$ is large enough.

Proof of the theorem. Assuming that Lemmas 2.2, 2.3 hold, the proof of the theorem may be obtained following the one for independent random variables, by

$$
\begin{aligned}
\sup & \left|\sqrt{D_{k}} P_{k}\left(\xi_{k}=p\right)-\exp \left(-\left(p-E_{k}\left(\xi_{k}\right)\right)^{2} / 2 D_{k}\right) / \sqrt{2 \pi}\right| \\
\leqq & \int_{-A}^{A}\left|E_{k}\left(\exp \left(i t \bar{\xi}_{k}\right)\right)-\exp \left(-t^{2} / 2\right)\right| d t \\
& \left.+\int_{|t| \geqq A} \exp \left(-t^{2} / 2\right) d t+\int_{A \leqq|t| \leqq \delta \sqrt{D_{k}}} \mid E_{k}\left(\exp i t \bar{\xi}_{k}\right)\right) \mid d t \\
& +\int_{\delta \sqrt{D_{k}} \leqq|t| \leqq \pi \sqrt{D_{k}}}\left|E_{k}\left(\exp \left(i t \bar{\xi}_{k}\right)\right)\right| d t
\end{aligned}
$$

If $A$ is large enough, the second term in the r.h.s. is small and, for every $A$, if the integral central limit theorem holds, the first term is also small, for large $k$. It is easily shown, using (2.1), (2.2), (2.3) and the assumptions (i), (ii) of (1.4) that $r_{0}, \delta$ may be chosen in such a way that the last two terms decrease to zero as $k$ increases. 


\section{Proofs}

In this section we will use a Mayer expansion to get the estimates (2.2), (2.3). The construction of the Mayer expansion, that is described in [4] for a gas of interacting particles in $\mathbb{R}^{v}$, has been carried out by various authors for systems similar to the one we will have to deal with $[5,6]$. We then only state in the following the main definitions and results that we will use, and refer to the literature for more details.

\subsection{Definitions}

Let $\mathscr{F}$ be a family of non-empty subsets $f \subset \mathbb{Z}^{v}$, consisting of at most two points. A polymer $R$ is a set $\left\{f_{1}, \ldots, f_{p}\right\}$ of distinct elements $f_{i} \in \mathscr{F}$, that is connected in the following sense: for any $f_{l}, f_{m} \in R$, there exist $f_{k_{1}}, \ldots, f_{k_{q}} \in R$ such that $f_{k_{1}}=f_{l}, f_{k_{q}}=f_{m}$ and $f_{k_{j}} \cap f_{k_{j+1}} \neq \varnothing$. Let $\mathscr{R}$ be the set of all polymers and, if $R \in \mathscr{R}$, denote by $\tilde{R}$ the subset of $\mathbb{Z}^{\nu}, \tilde{R}=\bigcup_{f \in R} f$; the activity $\zeta$ is a function $\zeta: \mathscr{R} \rightarrow \mathbb{C}$, such that $|\zeta(R)|$ is bounded and the partition function for a gas of polymers, with activity $\zeta$, and hard core interaction, in a subset $\Lambda \subset \mathbb{Z}^{v}$, is defined as:

$$
\Xi_{\Lambda}(\zeta)=1+\sum_{n \geqq 1} \sum_{\substack{\left\{R_{1}, \ldots, R_{n\}}\right\} \\ \tilde{R}_{i} \subset \Lambda, \mathbb{R}_{i} \cap \mathbb{R}_{j}=\phi, i \neq j}} \prod_{i=1}^{n} \zeta\left(R_{i}\right)
$$

We state now the main properties of the "low activity" expansion of the partition function (3.1).

3.2. Theorem. Let $\psi$ be a real, positive function on $\mathbb{Z}^{v}$ such that $\psi(0)=1$, $\sum_{x \in \mathbb{Z}^{v}}(\psi(x))^{1 / 2}=K<\infty$ and $z_{0}$ a positive number such that $\sqrt{z_{0}} K<1$. Assume that :

$$
|\zeta(R)| \leqq \prod_{f \in R} z_{0} \tilde{\psi}(f), \quad \tilde{\psi}(f)=\sup _{x, y \in f} \psi(x-y)
$$

Define on $\mathscr{P}=\bigcup_{n \geqq 1} \mathscr{R}^{n}$ the real function $\varphi^{T}$ as

$$
\varphi^{T}\left(R_{1}, \ldots, R_{n}\right)=\sum_{g \in \mathscr{G}_{n}\left(R_{1}, \ldots, R_{n}\right)}(-1)^{\# \text { edges in } g / n !}
$$

where $\mathscr{G}_{n}\left(R_{1}, \ldots, R_{n}\right)$ is the set of connected graphs with $n$ vertices $(1, \ldots, n)$ and edges $(i, j)$ corresponding to pairs $R_{i}, R_{j}$ such that $\widetilde{R}_{i} \cap \widetilde{R}_{j} \neq \varnothing$. We set the sum equal to zero if $\mathscr{G}_{n}$ is empty and one if $n=1$. Then

$$
\sum_{R . \tilde{R} \ni x}|\zeta(R)| \leqq B\left(z_{0}, K\right)
$$

and, if $z_{0} \exp \left(B\left(\sqrt{z_{0}}, K\right)\right)=C\left(z_{0}, K\right)<1$

$$
\Xi_{\Lambda}(\zeta)=\exp \left(\sum_{n \geqq 1} \sum_{\substack{\left(R_{1}, \ldots, R_{n}\right) \\ \widetilde{R}_{i} \subset A}} \varphi^{T}\left(R_{1}, \ldots, R_{n}\right) \prod_{i=1}^{n} \zeta\left(R_{i}\right)\right)
$$




$$
\begin{aligned}
& \sum_{n \geqq 1} \sum_{\substack{\left.R_{1}, \ldots, R_{n}\right) . \\
\exists R_{i}=R}}\left|\varphi^{T}\left(R_{1}, \ldots, R_{n}\right)\right| \prod_{i=1}^{n}\left|\zeta\left(R_{i}\right)\right| \\
& \quad \leqq \frac{\zeta(R)}{\prod_{f \in R} \sqrt{z_{0}}} C\left(z_{0}, K\right) /\left(1-C\left(z_{0}, K\right)\right)
\end{aligned}
$$

Proof. (3.4) and (3.5) may be deduced with standard methods (see for example the proof of 4.32 in [5]) if (3.3) holds. The absolute convergence of the series (3.4) can be derived from (3.5). We then prove only (3.3), that is the main "model dependent" statement of the theorem.

Let $\mathscr{T}_{x}$ be the set of walks on $\mathbb{Z}^{v}$, starting from $x$, that is

$$
\mathscr{T}_{x}=\left\{\left(x_{i}, \ldots, x_{n}\right), \quad n \geqq 1, \quad x_{i} \in \mathbb{Z}^{v}, \quad x_{0}=x\right\} .
$$

It is easy to check, by induction on the number of elements in $R$, that to every $R$, $x \in \tilde{R}$, may be associated a non-void subset $T_{x}(R) \subset \mathscr{T}_{x}$ such that the walk $\left(x_{0}, \ldots, x_{L}\right) \in T_{x}(R)$ if $\forall i=0, \ldots, L-1,\left\{x_{i}, x_{i+1}\right\} \in R$ and if for each two point set $f \in R$ there are exactly two indeces $0 \leqq i<j \leqq L-1$ such that $\left\{x_{i}, x_{i+1}\right\}=$ $\left\{x_{j}, x_{j+1}\right\}=f$ and for each one point set $f \in R$ there is exactly one index $0 \leqq i \leqq L-1$ such that $\left\{x_{i}\right\}=\left\{x_{i+1}\right\}=f$. It is clear that if $R \neq R^{\prime}, T_{x}(R) \cap T_{x}\left(R^{\prime}\right)=\varnothing$. Then if $t: \mathbb{Z}^{v} \times \mathbb{Z}^{v} \rightarrow \mathbb{R}$ is defined as $t(x, x)=z_{0}, t(x, y)=\left(z_{0} \psi(x-y)\right)^{1 / 2}, x \neq y$ :

$$
\begin{aligned}
\sum_{R . \tilde{R} \ni x} \prod_{f \in R} z_{0} \tilde{\psi}(f) & \leqq \sum_{\left(x_{0}, \ldots, x_{n}\right) \in \mathscr{T}_{x}} \prod_{i=0}^{n-1} t\left(x_{i}, x_{i+1}\right) \\
& \leqq \frac{\sqrt{z_{0}} K}{1-\sqrt{z_{0}} K}=B\left(z_{0}, K\right)
\end{aligned}
$$

We will also make use use of the following:

3.3. Proposition. Let $x \in \tilde{\Lambda}_{k}$, and $p_{x}\left(s \mid s_{\mathbb{Z}^{\vee} \backslash A_{k}}\right)$ be the probability distribution on the values of the spin in $x$ :

$$
p_{x}\left(s \mid s_{\mathbb{Z}^{v} \backslash \tilde{\hat{A}_{k}}}\right)=\exp \left(h_{x}\left(s \mid s_{\mathbb{Z}^{v} \backslash \tilde{\hat{A}_{k}}}\right)\right) / \sum_{s=n}^{m} \exp \left(h_{x}\left(s \mid s_{\overline{\mathbb{Z}}^{v} \backslash \hat{\hat{A}_{k}}}\right)\right)
$$

and denote with $E_{x}$ the expectation with respect to $p_{x}$. Then there exists a positive constant $d$ such that

(i) $E_{x}\left(\left(s-E_{x}(s)\right)^{2}\right) \geqq d$

and, for every $0<\delta<\pi$, there exists a strictly positive constant $c$ such that:

(ii) $\left|E_{x}(\exp (i t s))\right|<\exp (-c)$ for $\delta \leqq|t| \leqq \pi$

uniformly with respect to $S_{\mathbb{Z} v \mid \tilde{A}_{k}}$.

Proposition 3.3 is a direct consequence of the uniform lower bound:

$$
p_{x}\left(s \mid s_{\mathbb{Z}^{v} \backslash \tilde{A}_{k}}\right) \geqq \exp \left(-2 \sigma^{2}\|J\|\right) /|m-n| \forall s_{\mathbb{Z}^{v} \mid \tilde{A}_{k}}
$$

where $\|J\|=\sum_{t \in \mathbb{Z}^{v}}|J(t)|$. 
We proceed now to prove the lemmas. We start from the identity:

$$
\tilde{E}_{k}\left(\exp \left(i t \sum_{x \in \tilde{\Lambda}_{k}}\left(s_{x}-E_{x}\left(s_{x}\right)\right) / \sqrt{D_{k}}\right) \mid s_{\Lambda_{k} \backslash \tilde{\Lambda}_{k}}\right)=Z_{\tilde{\Lambda}_{k}}\left(h^{t}, \tilde{J}\right) / Z_{\tilde{\Lambda}_{k}}\left(h^{0}, \tilde{J}\right)
$$

that follows from (1.2) and the definition of $\tilde{E}_{k}(\cdot \mid \cdot) ; \tilde{J}$ is the restriction to $\mathbb{Z}^{v}\left(r_{0}\right)$ of $J$, and:

$$
h_{x}^{t}\left(s_{x} \mid s_{\mathbb{Z}^{v} \backslash \tilde{\Lambda}_{k}}\right)=h_{x}\left(s_{x} \mid s_{\mathbb{Z}^{v} \backslash \tilde{\Lambda_{k}}}\right)+i t\left(s_{x}-E_{x}\left(s_{x}\right)\right) / \sqrt{D_{k}}
$$

Introducing now suitable "polymer models", we will get estimates of the ratio in (3.8) by low activity expansions, as a consequence of theorem (3.2).

Proof of Lemma 2.2. Let $\mathscr{F}$ be the family of all the non-empty subsets of $\mathbb{Z}^{v}\left(r_{0}\right)$, with at most two points, and let $\psi_{f}$ be the function on the spin configurations in $f$ defined as:

$$
\begin{aligned}
& \psi_{\{x\}}\left(s_{x}\right)=\exp \left(i t\left(s_{x}-E_{x}\left(s_{x}\right)\right) / \sqrt{D_{k}}\right)-1 \\
& \psi_{\{x, y\}}\left(s_{x}, s_{y}\right)=\exp J(x-y) s_{x} s_{y}-1
\end{aligned}
$$

Define on the set of polymers $\mathscr{R}$ constructed by $\mathscr{F}$ the activity function:

$$
\zeta_{t}(R)=\sum_{\mid s_{\tilde{R}}} \prod_{x \in \tilde{R}} p_{x}\left(s_{x} \mid s_{\mathbb{Z} v_{\Lambda}}\right) \sum_{f \in R} \psi_{f}\left(s_{f}\right)
$$

where $p_{x}(\cdot \mid \cdot)$ is the probability distribution (3.7). Using the inequalities $\left|\psi_{\{x\}}(s)\right| \leqq$ $2 \sigma \delta$ for $|t|<\delta \sqrt{D_{k}},\left|\psi_{\{x, y\}}\left(s_{x}, s_{y}\right)\right| \leqq \sigma^{2}|J(x-y)| \exp \left(J_{r_{0}} \sigma^{2}\right)$ where $J_{r_{0}}=$ $\sup |\tilde{J}(t)|$, and assumption (1.1) on the pair potential, it is easily seen that, if $|t| \geqq r_{0}$

$r_{0}^{-1}$ and $\delta$ are small enough, the polymer model just defined satisfies the hypotheses of theorem 3.2 (choose for example $z_{0}=\max \left\{2 \sigma \delta, \sigma^{2} J_{r_{0}} e^{J_{r_{0}} \sigma^{2}}\right\} \psi(t)=|\tilde{J}(t)| / J_{r_{0}}$, $t \neq 0 ; \psi(0)=1)$ and that it is such that:

$$
Z_{\tilde{\Lambda}_{k}}\left(h^{t}, \tilde{J}\right)=\prod_{x \in \tilde{\Lambda}_{k}}\left(\sum_{s=n}^{m} \exp \left(h_{x}\left(s \mid s_{\mathbb{Z}^{\nu} \mid \tilde{\Lambda}_{k}}\right)\right) \Xi_{\tilde{\Lambda}_{k}}\left(\zeta_{t}\right)\right.
$$

The statement (3.4) of Theorem (3.2) gives then:

$$
\begin{aligned}
& \tilde{E}_{k}\left(\exp \left(i t \sum_{x \in \Lambda_{k}}\left(s_{k}-E_{x}\left(s_{k}\right)\right) / \sqrt{D_{k}}\right) \mid s_{\Lambda_{k} \backslash \tilde{\Lambda}_{k}}\right) \\
& =\Xi_{\tilde{\Lambda}_{k}}\left(\zeta_{t}\right) / \Xi_{\tilde{\Lambda}_{k}}\left(\zeta_{0}\right) \\
& =\exp \left(\sum_{n \geqq 1} \sum_{\substack{\left(R_{1}, \ldots, R_{n}\right) \\
\tilde{R}_{i} \subset \bar{\Lambda}_{k}}} \varphi^{T}\left(R_{1}, \ldots, R_{n}\right)\left[\prod_{i=1}^{n} \zeta_{t}\left(R_{i}\right)-\prod_{i=1}^{n} \zeta_{0}\left(R_{i}\right)\right]\right)
\end{aligned}
$$

Expanding the exponent in series of $t$, we get, for a suitable $\theta, \theta<\delta \sqrt{D_{k}}$

$$
\begin{aligned}
& \left|\exp \left(\sum_{n \geqq 1} \sum_{\substack{\left(R_{1}, \ldots, R_{n}\right) \\
\mathbb{R}_{i} \subset \boldsymbol{A}_{k}}} \varphi^{T}\left(R_{1}, \ldots, R_{n}\right)\left[\prod_{i=1}^{n} \zeta_{t}\left(R_{i}\right)-\prod_{i=1}^{n} \zeta_{0}\left(R_{i}\right)\right]\right)\right| \\
& \quad \leqq \exp \left(\frac{t^{2}}{2} \sum_{n \geqq 1} \sum_{\substack{\left(R_{1}, \ldots, R_{n}\right) \\
\widetilde{\boldsymbol{R}}_{i} \subset \tilde{\boldsymbol{A}}_{k}}} \varphi^{T}\left(R_{1}, \ldots, R_{n}\right) \operatorname{Re}\left[\left.\frac{d^{2}}{d t^{2}} \prod_{i=1}^{n} \zeta_{t}\left(R_{i}\right)\right|_{t=\theta}\right]\right)
\end{aligned}
$$




$$
\begin{aligned}
& \leqq \exp \left(\left.\frac{t^{2}}{2} \sum_{\substack{R .|\tilde{R}|=1 \\
\tilde{R} \in \tilde{\Lambda}_{k}}} \varphi^{T}(R) \operatorname{Re} \frac{d^{2}}{d t^{2}} \zeta_{t}(R)\right|_{t=\theta}\right) \\
& \cdot \exp \left(\frac{t^{2}}{2} \sum_{\substack{n, R_{1}, \ldots, R_{n} \\
\tilde{\boldsymbol{R}}_{i} \subset \tilde{\Lambda}_{k}}}^{\sum^{*}}\left|\varphi^{T}\left(R_{1}, \ldots, R_{n}\right)\right|\left|\frac{d^{2}}{d t^{2}} \prod_{i=1}^{n} \zeta_{t}\left(R_{i}\right)\right|_{t=\theta}\right)
\end{aligned}
$$

where $\sum^{*}$ ranges over $n$-ples $R_{1}, \ldots, R_{n}$ such that $\sum_{i=1}^{n}\left|\tilde{R}_{i}\right| \geqq 2$. Observe now that if $\tilde{R}=x, \varphi^{T}(R)=1 \zeta_{t}(R)=E_{x}\left(\exp \left(i t\left(s_{x}-E_{x}\left(s_{k}\right)\right) / \sqrt{D_{k}}\right)-1\right)$ so that the first factor in (3.14) may be estimated using Proposition (3.3) by:

$$
\sum_{x \in \tilde{\Lambda}_{k}} \operatorname{Re} \frac{d^{2}}{d t^{2}} E_{x}\left(i t\left(s_{x}-E_{x}\left(s_{x}\right) / \sqrt{D_{k}}\right)-1\right) \leqq-\left|\tilde{\Lambda}_{k}\right|(d+\varphi(\delta)) / D_{k}
$$

where $|\varphi(\delta)| \leqq 16 \sigma^{4} \delta^{2}$. The terms appearing in $\Sigma^{*}$ with $\mathrm{n}=2$ and $\tilde{R}_{1}=\tilde{R}_{2}=x$ give a contribution equal to $C \varphi(\delta)$ where $C$ is a constant not depending on $r_{0}, \delta, k$. This can be obtained easily using that $\left|\zeta_{t}^{\prime}(R)\right| \leqq$ const $\delta / \sqrt{D_{k}}$, if $|\tilde{R}|=1$. The remaining terms may be estimated using again the theorem 3.1. A direct computation gives in fact:

$$
\left|\operatorname{Re} \frac{d^{2}}{d t^{2}} \prod_{i=1}^{n} \zeta_{t}\left(R_{i}\right)\right| \leqq\left(\sigma^{2} / D_{k}\right) \prod_{i=1}^{n} \prod_{f \in R_{\mathfrak{t}}} \tilde{z} \tilde{\psi}(f)
$$

if $|t| \leqq \delta \sqrt{D_{k}}, \sum^{n}\left|\tilde{R}_{i}\right| \geqq 2$, where $\tilde{\psi}$ can be chosen as above and $\tilde{z}$ is such that $N^{2} z_{0}^{N-2+K} \leqq \begin{gathered}i=1 \\ \tilde{z}^{N+K}\end{gathered}$ with $N \geqq 2, K \geqq 1$. It is easy to see that it is sufficient to take $\tilde{z}>2^{-2 / 3} \sqrt{z_{0}}$ if $z_{0}<1 / 16$. For $r_{0}^{-1}, \delta$ small enough one gets that:

$$
\begin{aligned}
& \mid \tilde{E}_{K}\left(\exp \left(i t \sum_{x \in \tilde{\Lambda}_{k}}\left(s_{x}-E_{x}\left(s_{x}\right)\right) / \sqrt{D_{k}}\right)\left|s_{\Lambda_{k} \backslash \tilde{\Lambda}_{k}}\right|\right. \\
& \leqq \exp \left(\frac { t ^ { 2 } } { 2 } \left[-(d+\varphi(\delta))\left|\tilde{\Lambda}_{k}\right| / D_{k}+\right.\right. \\
& \left.+\left(\sigma^{2} / D_{k}\right) \sum_{x \in \tilde{\Lambda}_{k}} \sum_{R \cdot \tilde{R} \ni x}\left(\prod_{f \in R} \sqrt{\tilde{z}} \tilde{\psi}(f)\right) C(\tilde{z}, K) /(1-C(\tilde{z}, K))\right) \\
& \leqq \exp \left(\frac{t^{2}}{2}\left(-d+\varphi\left(r_{0}, \delta\right)\right)\left|\tilde{\Lambda}_{k}\right| / D_{k}\right)
\end{aligned}
$$

where $\varphi\left(r_{0}, \delta\right) \rightarrow 0$ if $\delta \rightarrow 0, r_{0}^{-1} \rightarrow 0$ and the lemma is proven.

Proof of lemma 2.3. Let now $\mathscr{F}$ be the family two points subsets of $\mathbb{Z}^{v}\left(r_{0}\right)$, and let $\mathscr{R}$ denote the set of polymers constructed by $\mathscr{F}$.

Define on $\mathscr{R}$ the real activity function

$$
\eta_{c}(R)=e^{c|\tilde{R}|} \sum_{s \tilde{\mathbf{R}}} \prod_{x \in \tilde{R}} p_{x}\left(s_{x} \mid s_{\mathbb{Z}^{v} \backslash \tilde{\Lambda}_{k}}\right) \prod_{f \in \boldsymbol{R}} \psi_{f}\left(s_{f}\right)
$$

where $c$ is a positive constant, and $\psi_{\{x, y\}}$ is given by (3.10). It is easy to show that if $r_{0}$ is big enough, theorem 3.2 applies to the polymer model defined above (choose for example $z_{0}=J_{r_{0}} \sigma^{2} \exp \left(J_{r_{0}} \sigma^{2}+2 c\right)$, and $\psi$ as above). 
It is now easy to check that:

$$
\begin{aligned}
& Z_{\tilde{\Lambda}_{k}}\left(h^{0}, \tilde{J}\right)=\prod_{x \in \tilde{\Lambda}_{k}}\left(\sum_{s} p_{x}\left(s \mid s_{\mathbb{Z}^{v} \backslash \tilde{\Lambda}_{k}}\right)\right) \Xi_{\tilde{\Lambda}_{k}}\left(\eta_{0}\right) \\
& Z_{\tilde{\Lambda}_{k}}\left(h^{t}, \tilde{J}\right) \leqq \exp \left(-c\left|\tilde{\Lambda}_{k}\right|\right) \prod_{x \in \tilde{\Lambda}_{k}}\left(\sum_{s} p_{x}\left(s \mid s_{\mathbb{Z}^{v} \backslash \tilde{\Lambda}_{k}}\right)\right) \text {. } \\
& \left(\sum_{\substack{n, \boldsymbol{R}_{1}, \ldots, \tilde{R}_{n} \\
\tilde{\boldsymbol{R}}_{i} \subset \tilde{\boldsymbol{\Lambda}}_{k}, \boldsymbol{R}_{i} \cap \tilde{\boldsymbol{R}}_{j}=\phi i \neq j}}\left(\prod_{i=1}^{n}\left|\eta_{c}\left(R_{i}\right)\right|\right) \prod_{\substack{x \in \tilde{A}_{k} \backslash \bigcup_{i=1}^{n} \tilde{R}_{i} \\
\sin }} \exp (c)\left|E_{x}\left(\exp \left(i t s_{x} / \sqrt{D_{k}}\right)\right)\right|\right)
\end{aligned}
$$

then, if $c$ is such that Proposition (3.3) applies, one finds, for $\delta \leqq|t| / \sqrt{D_{k}} \leqq \pi$ :

$$
\left|\tilde{E}_{k}\left(\exp \left(i t \sum_{x \in \tilde{\Lambda}_{k}} s_{x} / \sqrt{D_{k}}\right) \mid s_{\mathbb{Z}^{\nu} \backslash \tilde{\Lambda}_{k}}\right)\right| \leqq \exp \left(-c\left|\tilde{\Lambda}_{k}\right|\right) \Xi_{\tilde{\Lambda}_{k}}\left(\left|\eta_{c}\right|\right) / \Xi_{\tilde{\Lambda}_{k}}\left(\eta_{0}\right)
$$

Using again (3.4), (3.5), the ratio in the r.h.s. can be estimated as:

$$
\Xi_{\tilde{\Lambda}_{k}}\left(\left|\eta_{c}\right|\right) / \Xi_{\tilde{\Lambda}_{k}}\left(\eta_{0}\right) \leqq \exp \left\{z\left|\tilde{\Lambda}_{k}\right| B\left(\sqrt{z_{0}}, K\right) C\left(z_{0}, K\right) /\left(1-C\left(z_{0}, K\right)\right)\right\}
$$

where, for fixed $c, z_{0} \rightarrow 0$ for $r_{0} \rightarrow \infty$, and the lemma is proven.

Acknowledgement. We are grateful for many discussions with G. Gallavotti.

\section{References}

1. Gnedenko, B. V. : The theory of probability. Moscow, Nauka 1965, Engl. Trans. MIR 1976.

2. Dobrushin, R. L., Tirozzi, B. : Comm. Math. Phys. 54, 173-192 (1977)

3. Campanino, M., Del Grosso, G., Tirozzi, B. : to appear in J. Math. Phys.

4. Ruelle, D. : Statistical mechanics. New York: Benjamin 1969

5. Gallavotti, G., Martin-Lof, A., Miracle-Sole, A. : Lecture Notes in Phys. 20, 162-202 (1971)

6. Kunz, H. : Comm. Math. Phys. 59, 53 (1978)

Communicated by E. Lieb

Received April 24, 1979 